\title{
The PV MPPT Control Strategy Based on Generalized Predictive Control
}

\author{
Ni Fuyin ${ }^{1,2}$, Li Zhengming ${ }^{*}, 1$ and Shen Lin $^{2}$ \\ ${ }^{1}$ School of Electrical and Information Engineering, Jiangsu University, Jiangsu Zhengjiang, 212013, P.R. China \\ ${ }^{2}$ School of Electrical and Information Engineering, Jiangsu University of Technology, Jiangsu Changzhou, 213001, P.R. \\ China
}

\begin{abstract}
The excursion of maximum power point always exists due to nonlinear and time-varying characteristics of photovoltaic (PV) generation. To solve this problem, the maximum power point tracking (MPPT) control strategy based on generalized predictive control (GPC) is put forward. Firstly, the theory and feature of PV cell is analyzed, and the MPPT control model is constituted based on Boost circuit. Then the MPPT algorithm based on GPC is illustrated from such sections as prediction model, rolling optimization, online identification and feedback correction, the algorithm steps are given as well. Besides, the MPPT simulations are conducted by the methods based on GPC and PID in comparison. The comparison results testify that GPC algorithm is faster and more accurate to track the maximum power point. GPC algorithm has the characteristics like stability and robustness, and can be applied to solar generation system.
\end{abstract}

Keywords: Boost circuit, generalized predictive control, MPPT, photovoltaic cell, PID.

\section{INTRODUCTION}

With the industrialized society transforming to lowcarbon society in the current world, developing green energy has become the research focus in all countries. Solar power generation has cleanable and renewable characteristics, which is recognized publicly as the most potential new energy technique. The source of solar generation is PV array, and the output power has a maximum power point in different external environment like solar irradiation, temperature, and variable load etc. But the nonlinearity and time-varying characteristics result in the excursion of maximum power point. The research centralized on maximum power point tracking (MPPT) is conducted extensively, and many achievements are obtained [1-12].

Most of MPPT control strategies are combinations algorithms considering some intelligent control algorithm, or some improved strategies based on the classical algorithm. Generally, the researches on PV MPPT control strategies are mainly classified as below: (1) Constant voltage method. This method is simple and effective, but the accuracy is not very high, and the power loss is relative much. (2) Conductance increment method $[3,4]$. This method avoids misjudgment like constant voltage method, and can output stable voltage when tracking solar irradiation. Its drawback is that sensor needs high precision and system response needs high speed. Therefore the hardware cost of inverter is higher than the other methods. (3) Perturbation and observation ( $\mathrm{P} \& \mathrm{O})$ method [5-11], this method is the most applied and studied for its good performance and simple operation. The PID method in P\&O is to feedback and correct deviation depending on of history error data. So the tracking precision and speed of response has contradiction to a certain degree, and the system cannot achieve the optimal control result.

In order to overcome the insufficient of PID method, MPPT improved method based on generalized predictive control (GPC) is proposed in the article. Firstly, PV cell theory and the equivalent circuit based on Boost circuit are to be analyzed, the maximum power express is to be deduced as well. Then the MPPT control system based on GPC is to be presented from the processes including prediction model, rolling optimization, and online feedback correction. Next, the optimum control algorithm is to be realized according to PV array dynamic feature. Eventually, the simulation comparison analysis is to be performed by Simulink between PID method and GPC method.

\section{ANALYSIS OF PV CELL THEORY}

The math model of PV cell is presented in Eq. (1) [12]

$I=I_{p h}-I_{o}\left\{\exp \left[\frac{q\left(U+I R_{s}\right)}{A K T}-1\right\}-\frac{U+I R_{s}}{R_{s h}}\right.$

where,

$\begin{array}{ll}I & \text { Photovoltaic current (A) } \\ I_{p h} & \text { Photo current (A) } \\ I_{o} & \text { Diode saturation current (A) } \\ A & \text { Idealist factor for a p-n junction } \\ K & \text { Boltzman's factor }\left(1.381 \times 10^{-23} \mathrm{~J} / \mathrm{K}\right)\end{array}$ 
$T \quad$ Temperature of a solar array $\left({ }^{\circ} \mathrm{C}\right)$

$R_{s} \quad$ Series resistance $(\Omega)$

$R_{\text {sh }} \quad$ Shunt resistance $(\Omega)$

$q \quad$ Charge of an electron $\left(1.602 \times 10^{-19} \mathrm{C}\right)$

$U \quad$ Photovoltaic voltage (V)

The engineering math model of PV cell in the practical application is expressed in Eq. (2)

$I=I_{s c}\left\{1-C_{1}\left[\exp \left(\frac{U}{C_{2} U_{o c}}\right)-1\right]\right\}$

where, $C_{1}=\left(1-\frac{I_{m}}{I_{s c}}\right) \exp \left(\frac{-U_{m}}{C_{2} U_{o c}}\right)$,

$C_{2}=\left(\frac{U_{m}}{U_{o c}}-1\right)\left[\ln \left(1-\frac{I_{m}}{I_{s c}}\right)\right]^{-1}$

$I_{s c} \quad$ Short-circuit current (A)

$U_{o c} \quad$ Open-circuit voltage $(\mathrm{V})$

$I_{\mathrm{m}} \quad$ Current at the maximum power point (A)

$U_{m} \quad$ Voltage at the maximum power point $(\mathrm{V})$

And the four technical parameters are provided in the standard condition by PV manufacturer.

\section{ANALYSIS OF THE BOOST EQUIVALENT CIRCUIT}

The solar inverter uses two-stage structure. The frontstage is Boost circuit, which realizes the functions of voltage-rise and MPPT. The back-stage is grid-connected inverter, which achieves the function of inverting-inversion and grid connection. The Boost schematic diagram is as below in Fig. (1).

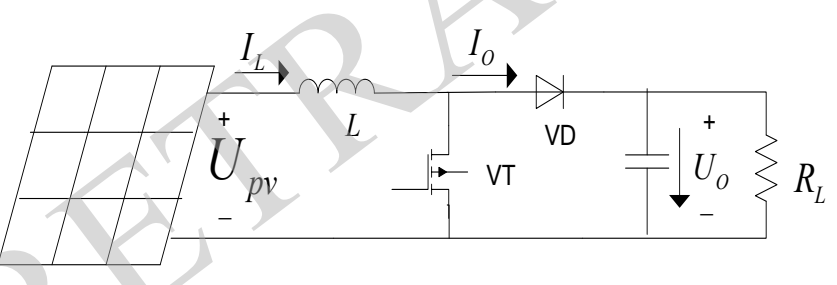

Fig. (1). Boost schematic diagram.

According Fig. (1), the relation between output voltage $U_{p v}$ and $U_{o}$ is:

$U_{p v} \cdot D \cdot T+\left(U_{p v}-U_{o}\right) \cdot(1-D \cdot T)=0$

where, $T$ is switching period of switching tube, $D$ is duty cycle, and its interval is $(0,1)$.

According to Eq. (3), the output voltage $U_{o}$ can be expressed in Eq. (4):

$U_{o}=\frac{1}{1-D} U_{p v}$

Equivalent transformation is employed in the Boost circuit, Fig. (1) is depicted as Fig. (2):

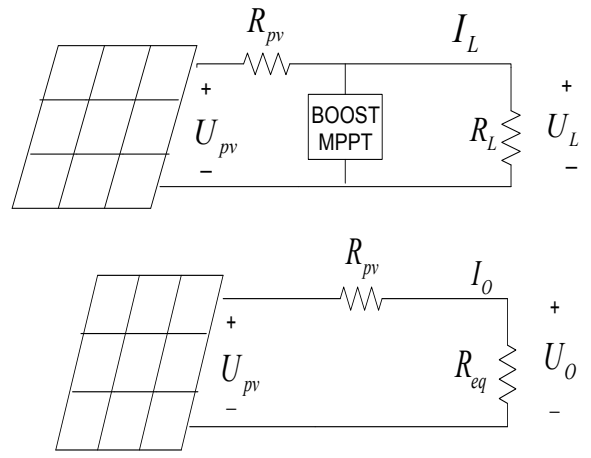

Fig. (2). Equivalent transformation of boost circuit.

where, $R_{p v}$ is the resistance of PV array. As shown in Fig. (2), the equivalent resistance is deduced in Eq. (5) in accord with Thevenin's theorem:

$R_{e q}=R_{L} \cdot(1-D)^{2}$

Therefore the value of input resistant is adjusted by means of regulating duty cycle of Boost circuit. When equivalent input impedance $R_{e q}$ is matched $R_{p v}$ by adjusting duty cycle, the PV array outputs maximum power expressed in Eq. (6):

$$
P_{\max }=\left(\frac{U_{P V}}{R_{P V}+R_{e q}}\right)^{2} \cdot R_{e q}=\frac{U_{P V}^{2}}{4 R_{e q}}
$$

\section{MPPT CONTROL SCHEME BASED ON GPC}

GPC has the characteristics such as predictive model, rolling optimization and feedback correction on line etc [13, 14]. So GPC fuses the advantages of predictive control and self-adaptive control, and easily estimates online parameters and realizes self-adaptive control. By means of rolling optimization, identification and feedback correction, GPC can estimate online model parameters according to the actual continuous input and output information. What's more, it can correct the control law to improve the control performance in different external environment.

The PV MPPT scheme based on GPC in Boost model is shown in Fig. (3). In the MPPT module, the P\&O method is adapt to output reference voltage $U_{r f}$ by the detection of PV array voltage and current output, and the $U_{r f}$ subtracts $U_{p v}$ to get the dynamic voltage error $U_{c r}$. GPC replaces traditional PID and regulate the error to output switch signal which is to control PWM module. Eventually, the output power of photovoltaic array is kept maximum according to the switching tube status controlled by PWM.

\section{GPC ALGORITHM IN MPPT STRATEGY}

\subsection{GPC Structure}

GPC algorithm structure is shown in Fig. (4) [15]. It takes the controlled auto-regressive moving average (CARIMA) as the predictive control model and describes the dynamic relation. Also, the identified model parameter and information are provided to the GPC optimization algorithm in real time. 


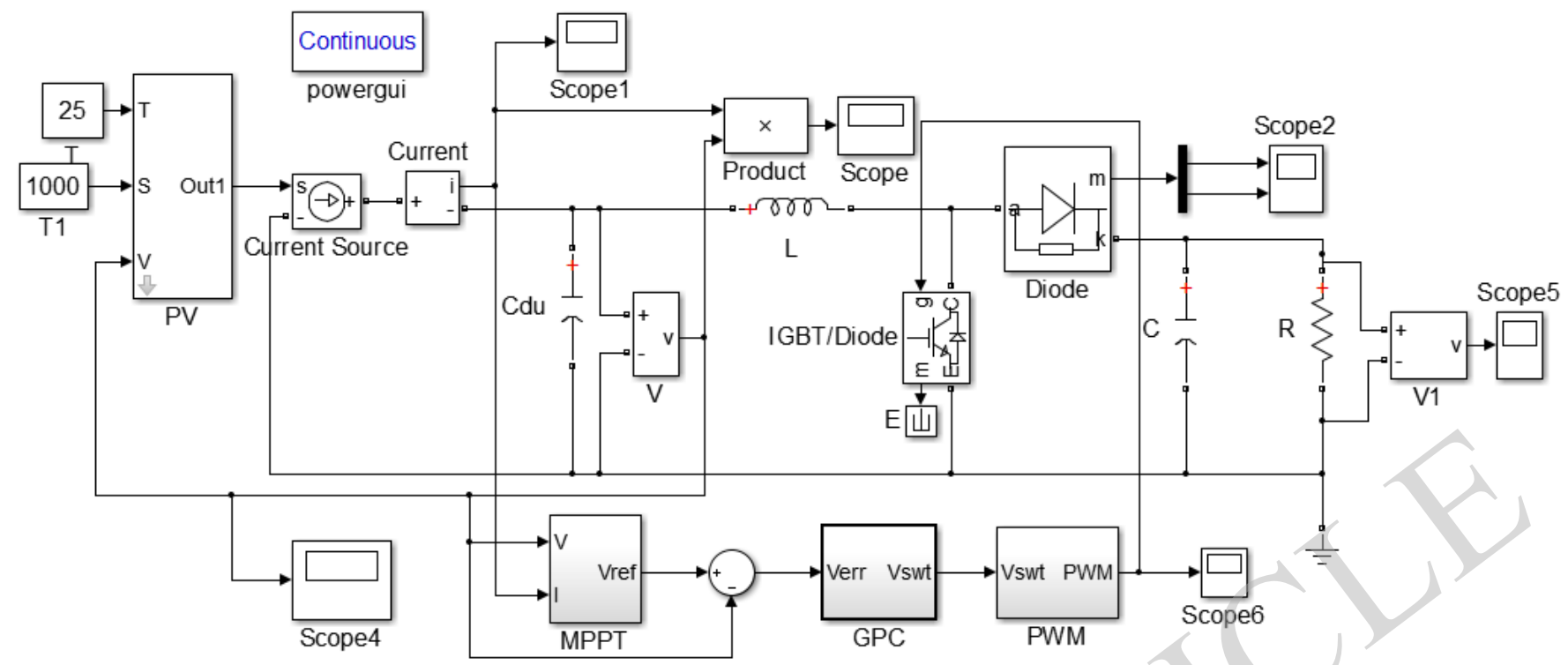

Fig. (3). PV MPPT control based on GPC in Boost modeling.

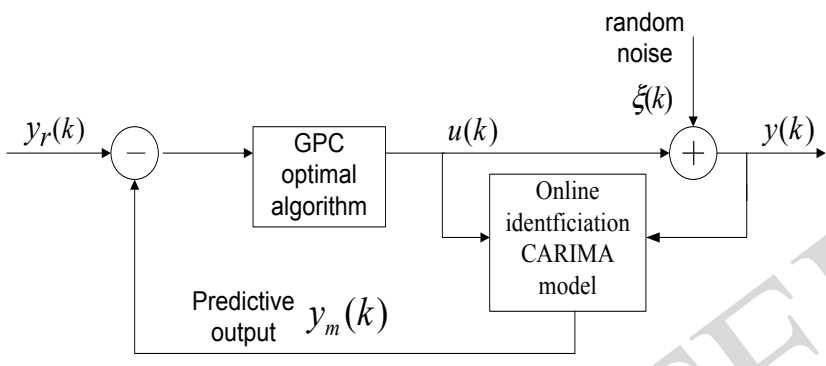

Fig. (4). GPC algorithm structure.

Then the reference voltage is calculated by the algorithm. $u(k)$ is input signal and equal to $U_{r e f}-U_{p v}$ in the Boost circuit. $y(k)$ is output signal, and equal to $V_{s w t}$ which control PWM, $y_{r}(k)$ is set-error of $U_{r e f}-U_{p v}$.
GPC control block diagram is shown in Fig. (5) [16]. It is composed of the process including softening, adjustment, and prediction. Note that the expected output vector is obtained after set-sequence is softened at every moment. Then the deviation vector is got after the expected output vector compared to the predictive output. The control increment $\Delta u(k)$ is got by dot product between deviation vector and dynamic vector $g^{T}$ at the moment. On one hand, the control increment acts on control object by the calculation of digital integration. On the other hand, it predicts new system output $f$ with the system output.

\subsection{Prediction Model}

CARIMA applied in the minimum variance control is used to describe basic prediction model of system dynamic

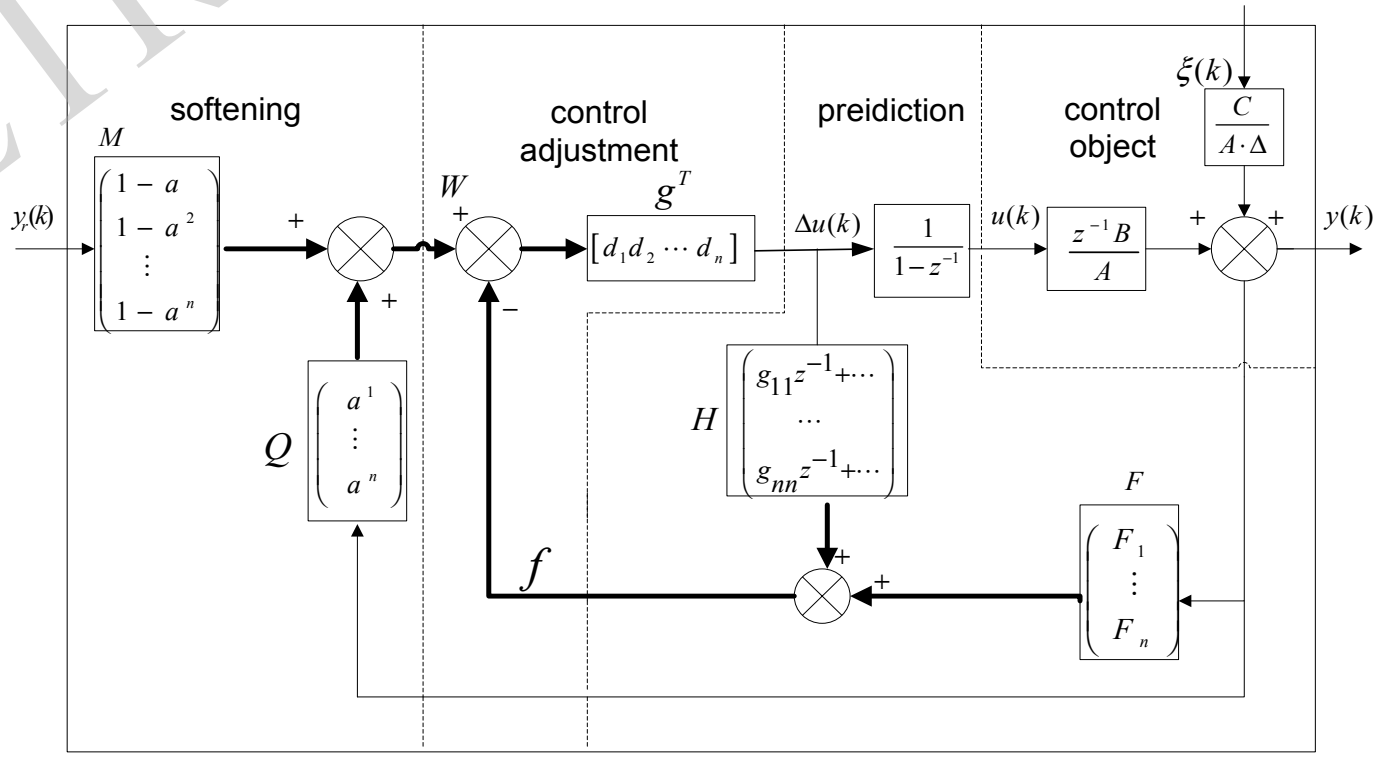

Fig. (5). GPC algorithm control block diagram. 
action. It has prediction function, and can predict future output according the history data and future input. The future system output is shown in Eq. (7):

$A\left(q^{-1}\right) y(k)=B\left(q^{-1}\right) u(k-1)+\frac{C\left(q^{-1}\right) \xi(k)}{\Delta}$

where, $A\left(q^{-1}\right)=1+a_{1} q^{-1}+\cdots+a_{n} q^{-n}$,

$B\left(q^{-1}\right)=b_{0}+b_{1} q^{-1}+\cdots+b_{m} q^{-m}$.

where, $q^{-1}$ is backward operator, $y(k) q^{-1}=y(k-1)$, $\Delta=1-q^{-1}$ is difference operator. $\xi(k)$ is the single random noise sequence that mean value is 0 . According to previous analysis, $u(k)$ and $y(k)$ is input and output of controlled object respectively. Note that $C\left(q^{-1}\right)=1$, and $n$ is order of input, and $m$ is order of output.

The system model at the $k+j$ moment is

$A\left(q^{-1}\right) y(k+j)=B\left(q^{-1}\right) u(k+j-1)+\frac{\xi(k+j)}{\Delta}$

For $y(k+j)$ exists unknown information, the Diophantine equation is introduced and the output predicted value is obtained at the $k+j$ moment. The Diophantine equation is presented in Eq. (9)

$1=A\left(q^{-1}\right) E_{j}\left(q^{-1}\right) \Delta+q^{-j} F_{j}\left(q^{-1}\right)$

where, $E_{j}\left(q^{-1}\right)=e_{j, 0}+e_{j, 1} q^{-1}+\cdots+e_{j, j-1} q^{-(j-1)}$,

$F_{j}\left(q^{-1}\right)=f_{j, 0}+f_{j, 1} q^{-1}+\cdots+f_{j, n} q^{-n}$.

$E_{j}$ and $F_{j}$ is determined by both $A\left(q^{-1}\right)$ and prediction length $j$. Then the simplified equation is got in Eq. (10):

$y(k+j)=B E_{j} \Delta u(k+j-1)+F_{j} y(k)+E_{j} \xi(k+j)$

Note that the future noise is unknown, but its mean is 0 . So GPC prediction model at the $k+j$ moment is

$y_{m}(k+j)=G_{j} \Delta u(k+j-1)+F_{j} y(k)$

where, $G_{j}=B E_{j}=\frac{B\left(1-q^{-j} F_{j}\right)}{A \Delta}=g_{j, 0}+g_{j, 1} q^{-1}+\cdots+g_{j, m+j-1} q^{-(m+j-1)}$.

\subsection{Rolling Optimization}

The optimization of prediction control is a kind of limited time domain rolling optimization, i.e., the optimized performance indicator involves the moment and a future limited time. Till the next moment the optimization period moves head. So the optimization is not conducted once time off line, but conducted continuously on line, that is so called rolling optimization.

GPC uses quadric performance indicator that the output error and the control increment are weighted: $\min J(k)=E\left\{\sum_{j=1}^{P}\left[y_{m}(k+1)-y_{r}(k+1)\right]^{2}+\sum_{j=1}^{M} \lambda_{j}[\Delta u(k+j-1)]^{2}\right\}$

where, $P$ is prediction time domain, $M$ is control time domain, $\lambda_{j}$ is control increment weighted parameter, which

is set to constant commonly. $y_{r}(k+j)$ is reference trajectory. Therefore, the expected output takes the form of reference trajectory so that the controlled object output $y(k)$ follows a specified soften curve. Eventually, $y_{r}(k)$ reaches the target $W$ smoothly.

Define:

$E_{j} B \Delta u(k+j-1)=G_{j 1} \Delta u_{1}+G_{j 2} \Delta u_{2}$

where, $\quad G_{j 1}=\left[g_{j, j-1} \cdots g_{j, 0}\right] \quad, \quad G_{j 2}=\left[g_{j, j+m-1} \cdots g_{j, j}\right]$,

$$
\Delta u_{1}=[\Delta u(k) \cdots \Delta u(k+j-1)]^{T},
$$$$
\Delta u_{2}=[\Delta u(k-m) \cdots \Delta u(k-1)]^{T} \text {. }
$$

According to Eq. (11) and (13), Eq. (14) is obtained:

$y_{m}(k+j)=G_{j 1} \Delta u_{1} G_{j 2} \Delta u_{2}+F_{j} y(k)$

Define: $G=\left[\begin{array}{c}G_{11} \\ G_{21} \\ \vdots \\ G_{P 1}\end{array}\right]=\left[\begin{array}{cccc}g_{1} & & & 0 \\ g_{2} & g_{1} & & \\ \vdots & & & \\ g_{M-1} & g_{M-1} & \ddots & g_{1} \\ \vdots & \vdots & \vdots & \vdots \\ g_{p} & g_{p-1} & \cdots & g_{P-M+1}\end{array}\right]$,

$f=\left[\begin{array}{c}G_{12} \Delta u_{2}+F_{1} y(k) \\ G_{22} \Delta u_{2}+F_{2} y(k) \\ \vdots \\ G_{P 2} \Delta u_{2}+F_{P} y(k)\end{array}\right]$

Considering control time domain defined as $M$,

Define:

$U=\Delta u_{1}=\left[\begin{array}{lll}\Delta(k) & \cdots & \Delta(k+j-1)\end{array}\right]^{T}=\left[\begin{array}{lll}\Delta(k) & \cdots \Delta(k+M-1)\end{array}\right]^{T}$

$Y_{m}=\left[y_{m}(k+1) \quad y_{m}(k+2) \quad \cdots \quad y_{m}(k+P)\right]$

where, $U$ is future controlled variable, $Y_{m}$ is future output. The model of prediction control is changed to vector form:

$Y_{m}=G U+f$

Also, Eq. (10) is changed to vector with Eq. (11) substituted into the equation, then Eq. (16) is got:

$J=\left(G U+f-Y_{r}\right)^{T}\left(G U+f-Y_{r}\right)+U^{T} U$

Calculate the derivative by the future variable $U$, set the results equals to 0 , the following express is obtained:

$U=\left(G^{T} G+\lambda I\right)^{-1} G^{T}\left(Y_{r}-f\right)$ 
where, $Y_{r}=\left[\begin{array}{lll}y_{r}(k+1) & y_{r}(k+2) \cdots y_{r}(k+P)\end{array}\right]$

The optimal controlled instant variable is

$\Delta u(k)=\left[\begin{array}{llll}1 & 0 & \cdots & 0\end{array}\right]\left(G^{T} G+\lambda I\right)^{-1} G^{T}\left(Y_{r}-f\right)$

Then, $u(k)=u(k-1)+\Delta u(k)$.

\subsection{Online Identification and Feedback Correction}

The future control effect is determined by optimization. The prediction control only takes effect at this moment, till the next moment, the control system checks the output of object, and corrects the prediction model control by the previous output, then begins the new optimization.

GPC control algorithm uses one control model, and keeps the prediction correctness by online feedback.

$\Delta y(k)$ in Eq. (9) is changed to Eq. (19):

$\Delta y(k)=(1-A) y(k)+B \Delta u(k-1)+\xi(k)$

And model parameter $(A, B)$ in Eq. (19) and data parameter $(y, u)$ is defined as vector $\theta_{k}$ and $\varphi_{k}$ respectively.

where, $\theta_{k}=\left[a_{1} \cdots a_{n} \mid b_{1} \cdots b_{n}\right]$

$\varphi_{k}=[\Delta y(k-1) \cdots-\Delta y(k-n) \mid-\Delta u(k-1) \cdots-\Delta u(k-m)]^{T}$

Therefore Eq. (16) is changed to :

$\Delta y(k)=\varphi_{k}^{T} \theta_{k}+\varepsilon(k)$

The recursive least-squares (RLS) method with forgetting factor is used to estimate model parameter $\theta_{k}$ :

$\theta(k)=\theta(k-1)+K(k)\left[y(k)-\varphi^{T}(k) \theta(k-1)\right]$

$K(k)=P(k-1) \varphi(k)\left[\varphi^{T}(k) P(k) \varphi(k)+\mu\right]^{-1}$

$P(k)=\frac{1}{\mu}\left[I-K(k) \varphi^{T}(k)\right] P(k-1)$

where, $\mu$ is forgetting factor, usually, $0.95<\mu \leq 1, K(k)$ is weight factor, $P(k)$ is positive definite covariance matrix. The initial value of parameter vector and covariance matrix should be set when to begin control, the common setting method is depicted in Eq. (24):

$\left\{\begin{array}{l}P(0)=\alpha^{2} I, \quad \alpha \text { is sufficently larege real number } \\ \theta(0)=\varepsilon, \quad \varepsilon \text { is sufficinently small real vector }\end{array}\right.$

In conclusion, the online operation step of GPC algorithm is as below:

(1) The parameter $n, m, P, M, \lambda, \mu$ and $\alpha$ is given in according with controlled object and control request of the Boost circuit.

Set $P(0)$ and $\theta(0)$ initial value, input preliminary data.

(3) Use RLS to estimate the model parameter $A\left(q^{-1}\right)$ and $B\left(q^{-1}\right)$, solve recursively Diophantine equation and get $F_{j}$ and $G_{j}$.
(6) Continue to circulation back to step (3).

\section{EXPERIMENT AND ANALYSIS}

\subsection{PV Array MPPT Simulation and Analysis at Different Time}

The photovoltaic Boost modeling is depicted in Fig. (6), the MPPT algorithm based on GPC is simulated by Simulink. The simulation parameters are as below:

$U_{o c}=22 \mathrm{~V}, I_{s c}=8.58 \mathrm{~A}, U_{m}=17.7 \mathrm{~V}, I_{m}=7.94 \mathrm{~A}, P_{\max }$ is about 140 W.

Comprehensive considering MPPT control effect after simulating some times, the parameters in GPC algorithm are given as below:

Calculation time period is $500 \mu \mathrm{s}$, control time domain $P=8$, prediction time domain $M=4$, weighted parameter $\lambda=1.2$, input order $n=2$, output order $m=3$, forgetting factor $\beta=0.99$.

Suppose the solar irradiation is $600 \mathrm{~W} / \mathrm{m}^{2}$, temperature is $20^{\circ} \mathrm{C}$. Note that the methods of MPPT are compared between PID and GPC, the $P_{\text {out }}$ output waves are shown in Fig. (6a, b) according to different control methods:

(a) $P_{\text {out }}$ by the method of PID

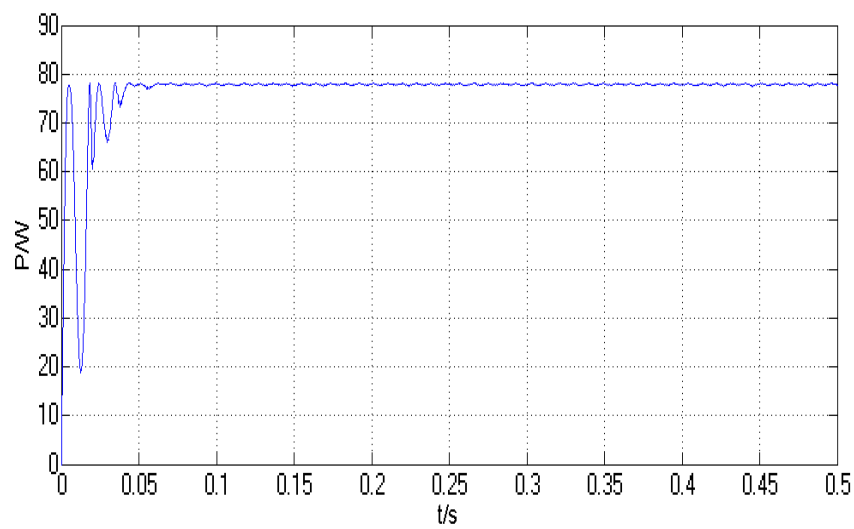

(b) $P_{\text {out }}$ by the method of GPC

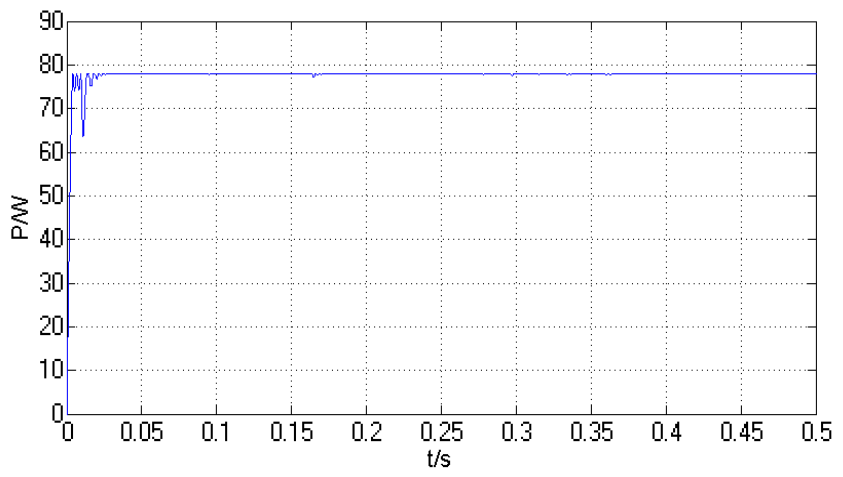

Fig. (6). MPPT control simulation when irradiation is $600 \mathrm{~W} / \mathrm{m}^{2}$. 
(a) $P_{\text {out }}$ by the method of PID

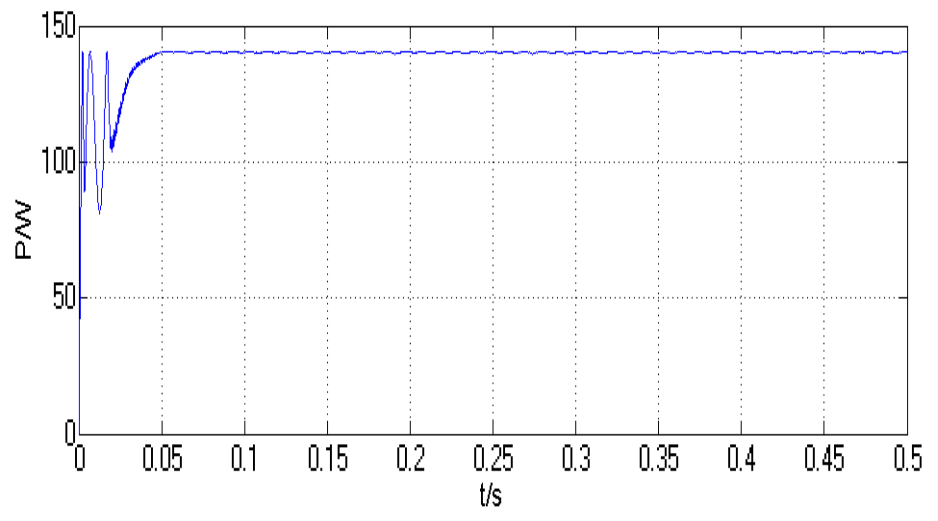

(b) $P_{\text {out }}$ by the method of GPC

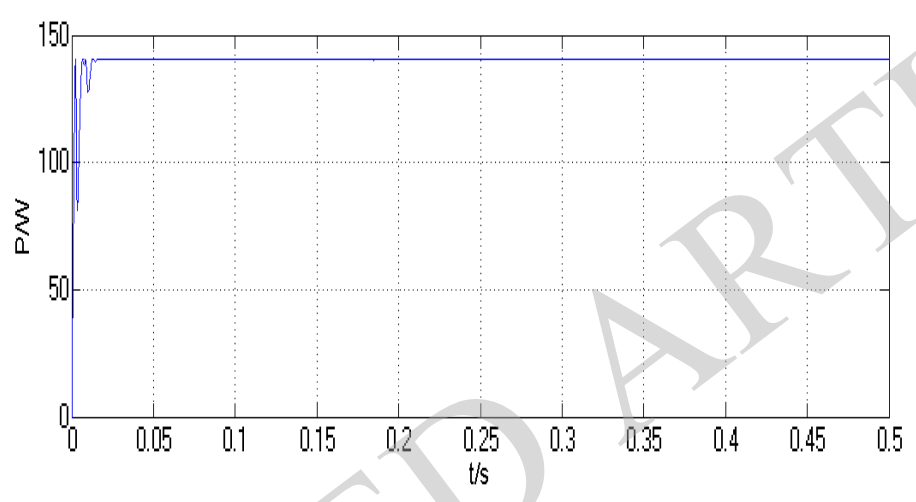

Fig. (7). MPPT control simulation when irradiation is $1000 \mathrm{w} / \mathrm{m}^{2}$.

(a) $P_{\text {out }}$ by the method of PID

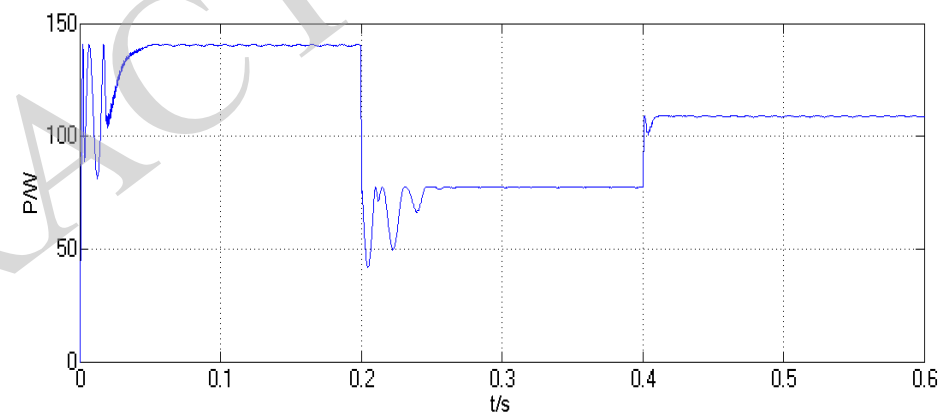

(b) $P_{\text {out }}$ by the method of GPC

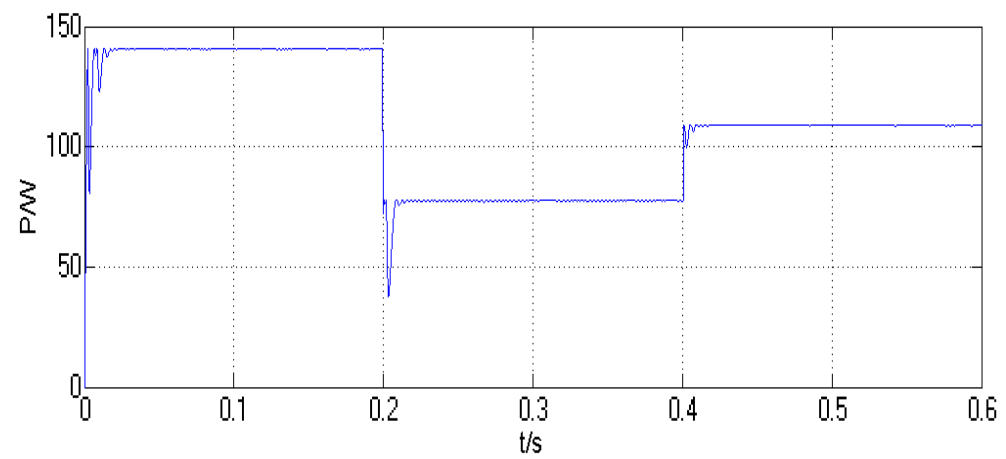

Fig. (8). MPPT simulation in the abrupt change of solar irradiation. 
Suppose the solar irradiation is $1000 \mathrm{~W} / \mathrm{m}^{2}$, temperature is $25^{\circ} \mathrm{C}$, the simulation figures are shown in Fig. $(\mathbf{8 a}, \mathbf{b})$ based on the method of PID and GPC:

From Fig. (7), it is concluded that the method on GPC is more accurate and faster to track the maximum power point than the method on PID. So GPC method decreases the loss and improves control stability and rapidity compared to PID method.

\subsection{MPPT Simulation in the Abrupt Change of Solar Irradiation}

The simulation is carried out to test the adaptive ability and dynamic response ability of MPPT algorithm by sudden change of solar irradiation. Note that the solar irradiation is separately set to $1000 \mathrm{~W} / \mathrm{m}^{2}$ in $0 \sim 0.2 \mathrm{~s}, 600 \mathrm{~W} / \mathrm{m}^{2}$ in $0.2 \sim 0.4 \mathrm{~s}$, and $800 \mathrm{~W} / \mathrm{m}^{2}$ in $0.4 \sim 0.6 \mathrm{~s}$. The MPPT simulation figures are shown in Fig. $(\mathbf{8 a}, \mathbf{b})$ by the methods of PID and GPC.

It is observed that the solar irradiation occurs sudden change at $0.2 \mathrm{~s}$ and $0.4 \mathrm{~s}$ from the dynamic response curve in Fig. (8). The method based on GPC can adjust maximum power point rapidly, and keep PV array output maximum power. GPC has better dynamic and stability feature, and improves control performance facing to various external environment at the comparison of PID. What's more, the GPC algorithm can keep high efficiency to track maximum power point and operate in the optimal status.

\section{CONCLUSION}

Aiming at the existing deviation of PV array maximum power point in different external environment, MPPT improved strategy based on GPC is proposed. The model of PV MPPT control based on GPC in Boost is made. Then the control express is deduced, the algorithm step is given as well. Next, the simulations between methods of GPC and PID control are given in different time and in the state of abrupt solar change respectively. The results indicate that GPC method shortens transient time clearly and increases disturbance-resisting ability, so the method improves the solar generation efficiency at the comparison of PID. Hence, the MPPT algorithm based on GPC provides a new strategy to solar generation research.

\section{CONFLICT OF INTEREST}

The authors confirm that this article content has no conflict of interest.

\section{ACKNOWLEDGEMENTS}

Supported by National Natural Science Foundation of China (51477070).

Supported by Jiangsu Province Prospective Joint Research Project (BY2015028-01).

Supported by Changzhou Key Laboratory of The Internet of Things New Technology and Application (CM20133008).

Supported by Natural Science Foundation of Jiangsu University of Technology (KYY14001).

Supported by A Project Funded by the Priority Academic Program Development of Jiangsu Higher Education Institutions (PAPD).

\section{REFERENCES}

[1] Sundareswaran, K.; Peddapati, S.; Palani, S. MPPT of PV systems under partial shaded conditions through a colony of flashing fireflies. IEEE Trans. Energy Conver., 2014, 29, 463-472.

[2] Mamarelis, E.; Petrone, G.; Spagnuolo, G. Design of a slidingmode controlled SEPIC for PV MPPT applications. IEEE Transactions on Industr. Electron., 2014, 61, 3387-3398.

[3] Hsieh, G.C.; Tsai, C.Y.; Wang, C.H. Photovoltaic power-increment $B$ aided incremental-conductance MPPT with two-phased tracking. IEEE Trans. Power Electro., 2013, 28, 2895-2911.

[4] Hsieh, G.C.; Hsieh, H.I.; Tsai, C.Y.; Wang, C.H. Photovoltaic power increment-aided incremental-conductance MPPT with twophased tracking. IEEE Trans. Power Electron., 2013, 28, 28952911.

[5] Jiang, Y.C.; Qahouq, J.A.A.; Haskew, T.A. Adaptive step size with adaptive-perturbation-frequency digital MPPT controller for a single-sensor. IEEE Trans. Power Electron., 2014, 28, 3195-3205.

[6] Lee, K.J.; Kim, R.Y. An adaptive maximum power point tracking scheme based on a variable scaling factor for photovoltaic. IEEE Trans. Energy Conver., 2012, 27, 1002-1008.

[7] Chen, Y.A.; Zhou, J.H.; Li, J.; Li, L.L. Application of gradient variable step size MPPT algorithm in photovoltaic system. Proc. CSEE, 2014, 34, 3156-3161.

[8] Kollimalla, S.K.; Mishra, M.K. A novel adaptive P\&O MPPT algorithm considering sudden changes in the irradiance. IEEE Trans. Energy Conver., 2014, 29, 602-610.

[9] Liu, S.J.; Zhuang, S.X.; Chen, X. Research on the control method of current disturbance observers for photovoltaic grid-connected inverters. Proc. CSEE, 2014, 33, 5811-5818.

[10] Sera, D.; Mathe, L.; Kerekes, T.; Spataru, S.V.; Teodorescu, R. On the perturb-and-observe and incremental conductance MPPT methods for PV systems. IEEE J. Photovoltaics, 2013, 3, 10701078.

[11] de Brito, M.A.G.; Galotto, L.; Sampaio, L.P.; de Azevedo e Melo, G.; Canesin, C.A. Evaluation of the main MPPT techniques for photovoltaic applications. IEEE Trans. Industr. Electron., 2013, 60, 1156-1167.

[12] Wang, H.B.; He, J.H. Application of conjugate gradient method in the photovoltaic system. J. Tsinghua Univ. (Sci. Technol.), 2014, $53,269-273$.

[13] Xu, F.; Wei, X.L.; Ren, L.H.; Luo, X.L. A control structure selection method based on multivariable generalized predictive control for unstable processes. Acta Automat. Sin., 2013, 39, 15471551. 
[14] Shi, W.G.; Shao, C.; Sun, Z.Y. Improved GPC network-control algorithm based on AR model time-delay prediction. Contr. Decision, 2012, 27, 477-480.

[15 ] Liu, Y.M.; Hua, Z.Y.; Chen, Y.Q. The application of GPC algorithm to the air-fuel ratio control of engine. Automot. Eng., 2013, 35, 403-407.
[16] Sun, J.; Zhang, H.Y.; Li, X.; Zhang D.H. Generalized predictive control scheme for monitor AGC system. J. Central South Univ. (Sci. Technol.), 2012, 43, 3852-3856.

Received: April 17, 2015

Revised: May 21, 2015

Accepted: May 26, 2015

(C) Fuyin et al.; Licensee Bentham Open.

This is an open access article licensed under the terms of the Creative Commons Attribution Non-Commercial License (http://creativecommons.org/licenses/by-nc/3.0/) which permits unrestricted, non-commercial use, distribution and reproduction in any medium, provided the work is properly cited. 